\title{
INVESTIGACIONES
}

\section{Habilidades sociales y comunicativas a través del arte en jóvenes con trastorno del espectro autista (TEA)*}

\author{
Social skills and communication skills through art in youth \\ with autism spectrum disorder (ASD)
}

\author{
Luz Coy Guerrero, ${ }^{a}$ Ernesto Martín Padilla ${ }^{b}$ \\ aniversidad Nacional Abierta y a Distancia, UNAD, Colombia \\ Correo electrónico: luz.coy@unad.edu.co \\ ${ }^{b}$ Universidad de la Sabana. Grupo de investigación Procesos psicológicos y contexto social \\ Correo electrónico: mario.martin1@unisabana.edu.co
}

\begin{abstract}
Esta investigación tuvo como objetivo establecer si es posible mejorar las habilidades comunicativas y la interacción social en jóvenes con trastorno del espectro autista (TEA), usando actividades artísticas. El método es etnográfico, longitudinal, con enfoque cualitativo. Se utilizó la técnica de observación participante, haciendo seguimiento durante talleres de arte. El estudio se desarrolló durante un año, haciendo cortes trimestrales de evaluación. La información se registró en diarios de campo, y se integró por medio de matrices comparativas y matrices globales, cotejando los resultados de los tres casos. Los participantes fueron tres estudiantes de una escuela de artes en Tunja, Colombia. Los resultados mostraron que los jóvenes mejoraron sus procesos de comunicación e interacción social gracias al aporte del arte como herramienta pedagógica. Se puede concluir que las técnicas derivadas del arte son útiles para fortalecer y mejorar las habilidades de comunicación y socialización en jóvenes con TEA.
\end{abstract}

Palabras clave: autismo, habilidades comunicativas, relaciones sociales, actividades artísticas, etnografía.

\begin{abstract}
This research aimed to establish if it is possible improving better communication skills and social interaction in young people with autism spectrum disorder (ASD) using art activities. We used the technique of participant observation through following up during art workshops. This is a longitudinal ethnographic method, with a qualitative approach. The study was conducted during a year, making quarterly cuts of evaluation. The information was registered in field diaries, and it was integrated through comparative arrays, and global arrays, comparing the results of the three cases. The participants were three students of an art school in Tunja, Colombia. The results showed that the young improved the communication process and the social interaction thanks to the contribution of the art as a pedagogic tool. It can be concluded that the techniques derived from art are useful to strengthen and improve communication and socialization skills in young people with ASD.
\end{abstract}

Key words: autism, communication skills, social relationships, art activities, ethnography.

Agradecemos a la Universidad de la Sabana por el apoyo en asesoría y fundamentación para el trabajo de investigación para grado de la Maestría en Psicología de la Salud y la Discapacidad, base para este artículo. Igualmente, se da un reconocimiento a la Escuela de artes CRE-SER-FUNAVI de la UNAD, por posibilitar el acceso a la muestra de jóvenes participantes. 
Estudios Pedagógicos XLIII, N $^{\circ}$ 2: 47-64, 2017

HABILIDADES SOCIALES Y COMUNICATIVAS A TRAVÉS DEL ARTE EN JÓVENES CON TRASTORNO DEL ESPECTRO AUTISTA (TEA)

\section{INTRODUCCIÓN}

Este artículo parte de un interés en abordar el proceso de interacción social de las personas con trastorno del espectro autista (TEA), lo cual ha representado un reto, tanto para los padres de los jóvenes TEA, como para sus otros cuidadores, debido a que uno de los principales impedimentos de las personas con trastorno de espectro autista (TEA) es la dificultad para comunicarse y expresar sus emociones (Miguel, 2006). Este trastorno abarca un gran abanico en la sintomatología entre los participantes, dado que el nivel de funcionamiento intelectual puede variar, encontrándose desde retraso mental profundo hasta una inteligencia normal o incluso alta en algunas áreas (Rodríguez \& Rodríguez, 2002). El recientemente elaborado sistema de clasificación DSM 5 reclasifica los trastornos generalizados del desarrollo por el Trastorno del Espectro Autista (TEA), eliminando todas las categorías diagnósticas que se incluían dentro de esta patología como entidades independientes, tales como el Síndrome de Asperger, el Síndrome de Rett, el Trastorno Desintegrativo de la infancia y el Trastorno Generalizado del Desarrollo no especificado (Morrison, 2015); todos estos cambios tratan de dar una mayor dimensionalidad al unirse las alteraciones sociales con las comunicativas "en un solo criterio definido como un déficit persistente en la comunicación social y la interacción social en distintos contextos" (Artigas-Pallares \& Paula, 2012, p. 584).

El término autista se introdujo por primera vez por Bleuler, en 1911, en alusión a la pérdida del contacto con la realidad y a estar ensimismado o encerrado en sí mismo (Cit. en Talero et al., 2003). Así, el autismo se entiende como un desorden espectro, lo cual significa que los síntomas, las características, pueden presentarse en una variedad amplia (o espectro) de combinaciones.

Las personas con TEA presentan un gran déficit en la percepción de las emociones, lo que constituye un elemento esencial para contribuir al desarrollo afectivo y emocional del sujeto (Miguel, 2006). Otros estudios refuerzan este tipo de versiones al ratificar que las personas con TEA presentan una compleja alteración a nivel neurobiológico, que incide en la interacción social recíproca del lenguaje y del comportamiento, lo cual se encuentra directamente relacionado con el lóbulo temporal, una de las áreas afectadas y a la que se le ha denominado cerebro social (Tager-Flusberhg, 1989). No obstante, de acuerdo con las investigaciones adelantadas en los últimos 20 años, a pesar de que las personas con TEA presentan déficits emocionales y cognitivos, se ha logrado comprobar que hay un desarrollo de habilidades de interacción y comunicación (Cuxart, 2000). En este sentido, se logró determinar que, según el grado de complejidad del trastorno generalizado del desarrollo, las personas con TEA podían desarrollar múltiples habilidades, respondiendo al grado de afectación neuronal de la persona, y podrían llegar a ser personas funcionales si se les direcciona su acción habitualmente mecánica y repetitiva (Organización Mundial de la Salud [OMS], 2006).

Por esta razón, la discusión sobre el TEA se ha centrado en estudiar el desarrollo de habilidades comunicativas e interacción social; específicamente, se ha querido establecer si un déficit cognitivo específico genera o no los síntomas sociales y comunicativos de los TEA (Baron-Cohen, 1990; Rajendran \& Mitchell, 2007). En este sentido, los déficit cognitivos que impactan en la alteración del lenguaje se constituyen en un importante factor para la presencia de síntomas en la expresión comunicativa del TEA (Joseph, TagerFlusberg \& Lord, 2002). 
Es importante resaltar que, en el proceso de interacción y comunicación de los jóvenes con TEA, estos presentan un rango que va desde la ausencia de conductas con intencionalidad comunicativa hasta el empleo de conductas de mayor complejidad funcional y formal; no obstante, en general, presentan menor riqueza comunicativa y pragmática en las dimensiones de expresión verbal, comprensión y conducta no verbal (Martos \& Ayuda, 2002); en casos más severos parecen receptores de información y con frecuencia sólo responden a solicitudes realizadas por sus padres o docentes (Brun \& Villanueva, 2004).

\subsection{DESARROLLO DE HABILIDADES DE COMUNICACIÓN}

La comunicación es esencial en la vida del ser humano. Mientras se avanza en el proceso de crecimiento, el individuo amplía progresivamente sus habilidades comunicativas en la medida en que crece su ámbito relacional. Es así como en un principio el mundo del recién nacido se limita a los padres y, en su defecto, a la persona encargada de su cuidado. Posteriormente empieza a incluir otros seres como miembros de la familia extensa, vecinos y otros niños cuando se integra a la vida escolar (López \& González, 2003).

En este sentido, los jóvenes con TEA presentan trastornos de interacción social recíproca, lenguaje y comportamiento, lo que sugiere que este síndrome es la expresión de un trastorno neurobiológico complejo, relacionado con la afectación de diversos circuitos neuronales; a la vez se presenta alteración a nivel del lóbulo temporal, estructura importante del denominado cerebro social, que muestra alteraciones morfológicas y funcionales en los sujetos con trastorno del espectro autista (García \& Peñas, 2009). Así, los trastornos del espectro autista (TEA) desde la temprana infancia no logran esta inclusión de las demás personas de su entorno por sus dificultades en la interacción social, en la cual puede haber desinterés por los demás y tendencia al aislamiento al no poder comprender las reglas y sutilezas sociales, y en la comunicación, al dificultarse el sostener una conversación recíproca por alteraciones en el lenguaje y pobreza en lo gestual y no verbal (Ministerio de Educación de Chile, 2010). Lo anterior justificaría la necesidad de utilizar actividades artísticas apropiadas de tipo visual y gestual para estimular el desarrollo de habilidades de comunicación e interacción social en las personas en condición TEA (Martín, 2009). El arte en cualquiera de sus disciplinas puede convertirse en una herramienta terapéutica y de inclusión para estos grupos poblacionales, ya que estimula la comunicación verbal y no verbal, promueve la integración e interacción efectiva en cada uno de los espacios sociales con sus semejantes y concatena satisfactoriamente la unidad psicofísica de estas personas de una manera armónica, y puede capacitar a una persona para cumplir un rol determinado en la sociedad, a partir de sus talentos, como se vio por ejemplo en un estudio sobre la utilización de recursos musicoterapéuticos (Benavides \& Orrego, 2010).

\section{MÉTODO}

La presente investigación se adelantó a través de un método cualitativo de tipo etnográfico y longitudinal, lo que permitió desarrollar descripciones detalladas de los cambios ocurridos en los procesos comunicativos y de interacción social de la población objeto de estudio, a partir de observaciones hechas a lo largo de un periodo de tiempo, en el medio donde se produce la experiencia vivida y relatada (Angrosino, 2012). La etnografía se 
realizó en el emplazamiento elegido, es decir, en el contexto educativo donde se aplicó la estrategia pedagógica, por parte del grupo investigador y docente, mediante la observación participante. Como criterio de fiabilidad, el método etnográfico usado de forma longitudinal permite hacer observaciones sistemáticas para corroborar de manera más consistente un patrón de conducta; estos patrones pueden ser cotejados por observadores múltiples, que al llegar a consensos confirman con más exactitud si han ocurrido los patrones y sus modificaciones (Angrosino, 2012).

\subsection{CATEGORÍAS DE ANÁLISIS}

\subsubsection{Desarrollo de habilidades de comunicación de los jóvenes con TEA}

Esta categoría describe las destrezas para iniciar o sostener una conversación, así como expresar sus ideas o necesidades con otras personas, aspectos comunicativos que representan una dificultad en el TEA (Morrison, 2015). Se evalúa la comunicación en su expresión y comprensión, tanto a nivel verbal (contenido y curso del habla, tono, volumen) como no verbal (gestos, expresiones corporales, atención) (Watzlawick, Beavin \& Jackson, 1986).

\subsubsection{Relaciones familiares y sociales de los TEA}

En esta categoría se describen y analizan las interacciones que se pueden detectar de los jóvenes con TEA con su núcleo familiar, así también el proceso de interacción que como seres individuales y sociales establecen con otras personas diferentes, quienes constituyen el núcleo social en el que se desenvuelven. Como componentes se evalúan la construcción de relaciones interpersonales funcionales y la integración e interacción efectivas.

\subsection{PARTICIPANTES}

Los participantes son tres jóvenes con espectro de autismo TEA, entre 25 y 27 años, pertenecientes al programa de artes de la Escuela CRE-SER-FUNAVI de la UNAD, que viven en Tunja, Colombia, de género masculino; no se tuvo en cuenta el estrato socioeconómico. Se escogieron intencionalmente por su diagnóstico; para el inicio de la observación se estableció el estado inicial de cada joven (Tabla 1).

\subsection{INSTRUMENTOS}

Los instrumentos de recolección de la información fueron la entrevista a padres, la descripción de la autobiografía de cada participante y el diario de campo. Para la estrategia pedagógica se realizaron talleres de arte.

\subsubsection{Entrevistas}

Las entrevistas implican que una persona calificada aplique una serie de preguntas a los sujetos participantes; estas son preguntas de sondeo, para lograr mayor productividad en la entrevista (Angrosino, 2012; Hernández, Fernández-Collado \& Batista, 2007). El guion de preguntas de sondeo, utilizado para la entrevista a padres fue el siguiente: 
1. ¿Presenta su hijo/a dificultades en la interacción social, como incapacidad para relacionarse con sus compañeros, falta de contacto ocular, pobre expresión facial, etc.? Especificar brevemente tales dificultades.

2. ¿Comparte su hijo/a objetos o intereses con otras personas, enseñando, mostrando o señalando?

3. ¿Manifiesta su hijo/sentimientos y emociones?; ¿entiende los nuestros?; ¿Hay reciprocidad social y comunicativa?

4. ¿Tiene su hijo/a lenguaje verbal?; si no lo tiene, ¿Lo compensa con el uso de gestos, mímica, etc.?; ¿Tiene ecolalias?

5. ¿Responde su hijo/a su nombre?; ¿Se gira cuando lo llama?

6. ¿Cómo es su lenguaje comprensivo?; ¿Es capaz de mantener conversaciones?; ¿Responde a órdenes sencillas?

7. ¿Cómo es el juego del alumno/a?; ¿Manipula objetos?; ¿Tiene juego de fícción o juego simbólico?

8. ¿Tiene estereotipias?

9. ¿Realiza actividades sin un fin aparente, por ejemplo, carreras sin sentido?

10. ¿Es inflexible?; ¿Le cuestan los cambios o las situaciones nuevas?; ¿Presenta rituales?

\subsubsection{Autobiografía de cada participante}

Las biografías, relatadas por los propios participantes, permitieron aportar datos para analizar la capacidad de comprensión y expresión verbal, así como la percepción de los participantes sobre sus relaciones familiares y sociales.

\subsubsection{Diario de campo}

El diario de campo es un instrumento que permite registrar, mediante la observación de los investigadores, aquellos hechos que son susceptibles de ser interpretados. En este sentido, el diario de campo es una herramienta que permite sistematizar las experiencias para luego analizar los resultados. Se utilizaron matrices para registro diario de los comportamientos de los jóvenes con TEA, así como los comentarios pertinentes, tanto de los jóvenes como de los padres que acudían a llevarlos a los talleres, sobre las categorías a registrar (Hernández et al., 2007).

\subsubsection{Talleres de arte}

Los talleres de arte, realizados por instructores entrenados en la institución, fueron los siguientes:

\section{Música}

Objetivo: Actualmente la musicoterapia se ha convertido en una disciplina de la salud, ya que facilita la relación y la comunicación de sentimientos y emociones a las personas que no tienen facilidad de expresión con su entorno (Betés del Toro, 2000).

La comprensión de la música implica por tanto el análisis secuencial y el procesamiento global (gestáltico) de la información con actividad probablemente bi-hemisférica, 
cooperando en ella el hemisferio izquierdo para componentes más analíticos y el derecho en los componentes más emocionales de forma más intensa que en el lenguaje hablado. (Barquero, 2008, p. 25).

Descripción de la actividad: El tallerista inicia su proceso terapéutico mediante la exposición de diferentes ritmos musicales, para detectar acciones y reacciones en los jóvenes con TEA, a fin de identificar el ritmo y la melodía de más agrado. Se establecen con estos ejercicios acciones y reacciones socio afectivas; estas respuestas se estimulan mediante la interacción, lo que permite evidenciar cambios importantes a nivel del bienestar emocional, la interacción social y la comunicación, además de permitir observar mejorías en los procesos de memoria y recordación. Esto se puede evidenciar durante la planeación y ejecución de una obra musical, pues los participantes deben lograr el aprendizaje de las obras musicales, así como mostrar interés y un uso adecuado de los instrumentos musicales asignados. Así, se incentiva el trabajo en equipo y su capacidad de comunicar y dar a los demás su producto, en la presentación de las obras musicales en eventos especiales.

Duración: este ejercicio se realizó durante un año, mediante una sesión semanal de 90 minutos.

\section{Danza}

Objetivo: Se promueve, a través de la danza, el contacto entre personas, el trabajo en equipo y la comunicación corporal, con el fin de mejorar procesos de interacción y comunicación social. Según Betés del Toro (2000), hay un enorme potencial en el movimiento pautado y direccionado en estos grupos poblacionales.

Descripción de la actividad: El tallerista inicia con una exploración del movimiento libre, como respuesta a los ritmos musicales que le está colocando en cada sesión de danza, paralelamente les comparte videos de danza, donde les permite ver a los niños que para bailar se necesitan dos o tres personas en el mismo escenario, ejercicio que se hace repetitivo dos o tres veces en la semana, con la intención de buscar en los jóvenes TEA un acercamiento y una respuesta paulatina.

Duración: ejercicio que se realizó durante un año, dos veces a la semana, mediante una sesión de 90 minutos.

\section{Títeres}

Objetivo: Según Russell (2000), históricamente el títere se utilizó como una técnica terapéutica del psicoanálisis, para tratar la neurosis infantil; la elaboración de un fenómeno comunicacional logrado por el títere sentó las bases para creer que este posee alguna cualidad esencial que permite superar barreras y llegar al "yo". El objetivo de utilizar los títeres como medio terapéutico es proporcionar un medio de interacción que estimule el aumento de expresiones y comprensiones de mensajes comunicacionales y sociales, lo que facilita la respuesta del Participante estimulando su lógica (Lions, s. f.).

Descripción de la actividad: El tallerista inicia una exploración de voces, mediante la asignación de nuevos amigos para los participantes, quienes le podrían colocar un nombre preferido, así estos títeres se convierten en la voz de sus amigos favoritos; los jóvenes con TEA inician su proceso de exploración del funcionamiento del títere. Posteriormente, el 
tallerista utiliza también una muestra de pequeñas fracciones de obras literarias, donde se evidencia la interacción entre los títeres.

Duración: el ejercicio se realizó durante un año, mediante una sesión semanal de 90 minutos.

\section{Pintura}

Objetivo: Según Epelde (2009), la técnica de pintura favorece el proceso terapéutico en el autismo, ya que la pintura tiene muchas ventajas. Al pintar, surgen de manera explícita los personajes que se han llevado interiormente. La pintura lleva a comprender nuestra particular forma de representar y de representarnos en el mundo.

Descripción de la actividad: El tallerista inicia esta técnica, animando a los jóvenes con TEA a que utilicen los materiales como papel, témperas y colores de forma libre, pues esta técnica le permite a este grupo poblacional plasmar la información contenida en su interior de forma no verbal, evidenciando con esto manifestaciones simbólicas, vivencias y expresiones, que no suelen exponer libremente, por su mismo cuadro patológico.

Duración: ejercicio que se realizó durante un año, mediante una sesión semanal de 90 minutos.

\subsection{PROCEDIMIENTO}

FASE 1: Definición de estado inicial: Se hizo una descripción funcional cognitiva (TEA) de las alteraciones cognitivas de los tres Participantes con trastorno espectro de autismo, a nivel de desórdenes neurobiológicos crónicos, caracterizados por un patrón de alteraciones en la reciprocidad social, la comunicación verbal y no verbal, y la flexibilidad a nivel conductual (Sperber \& Wilson, 1986). Para esto se tomó la historia clínica para el diagnóstico, se solicitó a cada joven participante su biografía y se aplicó el guion de entrevistas a los padres, para establecer las condiciones iniciales según las categorías establecidas y poder reportar así los cambios posteriores.

FASE 2: Talleres de arte: Se llevaron a cabo en las aulas de la escuela de artes. Los grupos en cada taller estaban compuestos por 18 estudiantes, de los cuales una parte tenía diagnóstico de trastornos diversos, (incluyendo los tres jóvenes del estudio), mientras que una parte de los jóvenes no tenía condiciones especiales. Estos jóvenes se incluyen habitualmente en los talleres de la fundación con el objeto de apoyar a los docentes en la planeación y ejecución de las actividades artísticas, para mantener el equilibrio grupal en la organización de las mismas; así, se puede hacer un manejo interno en los grupos de los síntomas de los estudiantes con TEA y otros trastornos.

FASE 3: Evaluación de los cambios: Se elaboró el diario de campo, que fue consignado en las matrices, donde se colocaron los cambios progresivos en cada caso por resultados de los talleres y categorías. El estudio se desarrolló durante un año, haciendo cortes trimestrales de evaluación. La información se registró en los diarios de campo, y se integró por medio de matrices comparativas y matrices globales, cotejando los resultados de los tres casos. 


\section{RESULTADOS}

\subsection{DESCRIPCIÓN DEL ESTADO INICIAL DE LOS PARTICIPANTES}

Se estableció el estado inicial obtenido por el reporte de los padres (entrevista) del Participante (autobiografía) y la descripción funcional consignada en el informe de ingreso (historia Clínica) (ver Tabla 1).

Tabla 1. Estado inicial de los participantes con TEA

\begin{tabular}{|c|c|c|}
\hline CASO & Categoría & Descripción \\
\hline \multirow{3}{*}{$\sum_{0}^{\infty}$} & $\begin{array}{l}\text { Descripción } \\
\text { Funcional y } \\
\text { cognitiva } \\
\text { (Historia } \\
\text { Clínica) }\end{array}$ & $\begin{array}{l}\text { Participante con TEA asociado a principio de esquizofrenia, que presenta } \\
\text { alteración en las funciones ejecutivas; denota además mutismo selectivo, no } \\
\text { interpreta las bromas, y presenta dificultad para ejecutar operaciones de lógica } \\
\text { matemática y disgrafía. También se observa aparente aislamiento e indiferencia } \\
\text { ante los demás niños, pasividad, y solo reacciona ante órdenes. Se comprueba } \\
\text { que él tiene buena capacidad de memoria y por lo mismo se le matricula a un } \\
\text { curso libre a nivel de pregrado en Ingeniería de Sistemas en la universidad, lo } \\
\text { cual ha dado excelente resultado, pues responde de forma adecuada y con gran } \\
\text { agilidad las lecciones evaluativas, con excelente puntaje a nivel académico y } \\
\text { mejorando a su vez el nivel de comunicación e interacción social. }\end{array}$ \\
\hline & $\begin{array}{l}\text { Categoría 1: } \\
\text { Desarrollo de } \\
\text { habilidades de } \\
\text { comunicación } \\
\text { (Entrevista a } \\
\text { padres) }\end{array}$ & $\begin{array}{l}\text { Muestra características de mutismo selectivo, aislamiento total, falta contacto } \\
\text { visual e interpersonal, escaso uso y comprensión de la información transmitida } \\
\text { por gestos. Presenta sensibilidad aguda a ciertos sonidos, así como voz alta y } \\
\text { estridente. }\end{array}$ \\
\hline & $\begin{array}{l}\text { Categoría 2: } \\
\text { Relaciones } \\
\text { familiares y } \\
\text { sociales } \\
\text { Entrevista a } \\
\text { padres/Auto- } \\
\text { biografía }\end{array}$ & $\begin{array}{l}\text { Familia de padres separados; vive con su madre, quien padece la misma patología } \\
\text { que él. Dice su madre que en ocasiones presenta conductas autodestructivas, } \\
\text { razón por la que permanece bastante medicado para poderlo controlar. Sin } \\
\text { embargo, su madre comenta que el que él est'r participando en actividades } \\
\text { artísticas le ha permitido mejorar su comportamiento habitual y de las relaciones } \\
\text { sociales. El joven relata problemas en las relaciones en la escuela: "tenía muchos } \\
\text { problemas con mis compañeros y con mis profesores porque siempre me } \\
\text { echaban la culpa de todo, me regañaban por culpa de los demás" En la familia: } \\
\text { "La relación con mi familia va bien aunque a veces tengo problemas con ellos". }\end{array}$ \\
\hline
\end{tabular}




\begin{tabular}{|c|c|c|}
\hline \multirow{3}{*}{ 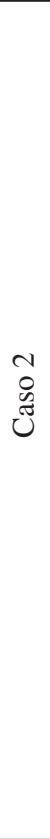 } & $\begin{array}{l}\text { Descripción } \\
\text { Funcional y } \\
\text { cognitiva } \\
\text { (Historia } \\
\text { Clínica) }\end{array}$ & $\begin{array}{l}\text { Participante con Síndrome de West, quien padece ataques epilépticos; hay } \\
\text { alteración en las funciones ejecutivas; trastorno pragmático del lenguaje, como } \\
\text { aspecto más típico de la alteración de la comunicación, y con tendencia al uso } \\
\text { repetitivo estereotipado y rígido de las palabras. Se evidencia atención dispersa, } \\
\text { lo cual genera problemas en la memoria de corto plazo, ya que no retiene ni } \\
\text { elabora la información entregada. Tiene escaso uso y comprensión de la } \\
\text { información, y no procesa adecuadamente la comunicación no verbal de otras } \\
\text { personas. }\end{array}$ \\
\hline & $\begin{array}{l}\text { Categoría 1: } \\
\text { Desarrollo de } \\
\text { habilidades de } \\
\text { comunicación } \\
\text { (Entrevista a } \\
\text { padres) }\end{array}$ & $\begin{array}{l}\text { Presenta conductas inapropiadas, uso repetitivo estereotipado y rígido de } \\
\text { palabras y frases, e incluso utiliza gestos para pedir algunas cosas. }\end{array}$ \\
\hline & $\begin{array}{l}\text { Categoría 2: } \\
\text { Relaciones } \\
\text { familiares y } \\
\text { sociales } \\
\text { Entrevista a } \\
\quad \text { padres/ } \\
\text { Autobiografía }\end{array}$ & $\begin{array}{l}\text { Participante que presenta una familia nuclear con permisividad excesiva, razón } \\
\text { por la cual suelen tener discusiones con sus padres, pues no hace caso, lo que ha } \\
\text { generado que el joven sea voluntarioso y rebelde. Su madre intenta corregirlo, } \\
\text { pero su padre la desautoriza, de manera que el joven siempre termina haciendo } \\
\text { lo que quiere. Como fortalezas sociales es un joven que manifiesta } \\
\text { permanentemente su efusividad y su alegría con sus padres; le gusta socializar } \\
\text { con todos los visitantes que llegan a su casa, aunque le gusta estar con ellos solo } \\
\text { en el comedor. El joven relata que antes de dedicarse al arte "me rechazaban de } \\
\text { todo lado porque era muy inquieto y por eso todos me miraban mal"; pero al } \\
\text { destacarse en la música y el estudio ha mejorado sus relaciones sociales. }\end{array}$ \\
\hline \multirow{3}{*}{ 必 } & $\begin{array}{l}\text { Descripción } \\
\text { Funcional y } \\
\text { cognitiva } \\
\text { (Historia } \\
\text { Clínica) }\end{array}$ & $\begin{array}{l}\text { Participante con TEA en una de las formas más leves de trastornos autísticos, } \\
\text { con alteración en las funciones ejecutivas, trastorno pragmático de lenguaje } \\
\text { comunicación y de interacción social, mutismo selectivo como aspecto más } \\
\text { típico de la alteración y dificultad en el aprendizaje debido al déficit marcado en } \\
\text { las áreas de comunicación y socialización, además de evidenciar ecolalia dado el } \\
\text { uso repetitivo y estereotipado y rígido de las palabras y frases; evidencia } \\
\text { problemas de comprensión de lenguaje, normas y rutinas diarias, lo cual genera } \\
\text { alteración de la conducta. No tiene lenguaje simbólico, su lenguaje es muy literal } \\
\text { y obsesivo; no interpreta metáforas y bromas y ríe cuando ve reír. El déficit } \\
\text { cognitivo y problemas de aprendizaje que presenta el participante no le han } \\
\text { permitido culminar su proceso de formación académica: está en básica, por lo } \\
\text { que aún sigue en aula multigrada; le gusta alinear los objetos, es rutinario. }\end{array}$ \\
\hline & $\begin{array}{l}\text { Categoría 1: } \\
\text { Desarrollo de } \\
\text { habilidades de } \\
\text { comunicación } \\
\text { (Entrevista a } \\
\text { padres) }\end{array}$ & $\begin{array}{l}\text { Presenta alteración a nivel cognitivo conductual, no establece contacto ocular, } \\
\text { escaso control de tono, volumen y entonación de voz. Participante que presenta } \\
\text { movimientos estereotipados, poca empatía y reciprocidad. }\end{array}$ \\
\hline & $\begin{array}{l}\text { Categoría 2: } \\
\text { Relaciones } \\
\text { familiares y } \\
\quad \text { sociales } \\
\text { Entrevista a } \\
\text { padres/Auto- } \\
\text { biografía }\end{array}$ & $\begin{array}{l}\text { Familia monoparental, vive con su madre. El joven permanece solo mucho } \\
\text { tiempo en casa; esto ha repercutido en repetidos conflictos con su madre, pues el } \\
\text { joven no atiende órdenes inmediatas de ella. Tiene dificultades para comprender } \\
\text { situaciones sociales, no entiende las bromas y se retraen ante un abrazo o beso; } \\
\text { bastante rígido, pues no permite que le cambien las cosas de donde las tiene; por } \\
\text { la misma razón es obsesionado por el orden. El joven relata que "mi mamá } \\
\text { siempre ha estado pendiente de mí; mi papá no se relaciona mucho conmigo, } \\
\text { solamente el saludo. Estuve en el colegio hasta noveno grado, soy muy serio, me } \\
\text { gusta la danza y el dibujo y soy feliz en la Escuela" Sin embargo dice que "No } \\
\text { me gustan las personas", lo que indica mayor interés por los objetos y actividades } \\
\text { que por las relaciones sociales. }\end{array}$ \\
\hline
\end{tabular}




\subsection{OBSERVACIONES SOBRE EL IMPACTO DE LAS ACTIVIDADES ARTÍSTICAS}

Las observaciones de los talleristas en cada una de las actividades artísticas desarrolladas durante el año se sintetizan así (ver Tabla 2):

\section{Música}

A lo largo de los talleres se pudo corroborar el efecto benéfico de la música en los tres jóvenes con TEA de este estudio, ya que mediante sus gustos musicales se integraron y aumentaron su participación en el trabajo grupal al montar diferentes obras musicales; igualmente, la música tuvo un efecto en las emociones, pues los calmaba cuando estaban ansiosos y los hace más alegres y proactivos al relacionarse con los demás compañeros del grupo. Se evidenció que la música les genera una alta motivación, lo cual ha generado muy buen resultado a nivel de interacción y comunicación entre los mismos y su entorno.

\section{Danza}

Los ejercicios de danza-movimiento lograron varios beneficios tales como mejoramiento de la autoimagen; desarrollo de la sensación kinestésica que ayuda en la movilidad, lateralidad y direccionalidad; incremento en la motivación; desarrollo de la conexión mente-cuerpo; desarrollo de la cooperación social y trabajo grupal.

Ahora bien, se observó que la danza fue uno de los métodos más eficaces para integrar socialmente a los jóvenes con TEA, pues con el paso de los meses se evidenció que los jóvenes con esta patología han progresado bastante a nivel de contacto visual, integración y manejo de relaciones interpersonales básicas, (saludo y compartir), y han mejorado notablemente en sus procesos de interacción y comunicación social con su entorno familiar y escolar.

\section{Títeres}

En los tres casos se avanzó en que estos jóvenes participaran de forma proactiva a través de sus títeres, que se convirtieron en sus amigos imaginarios; a través de estos se mejoró en la expresión verbal y no verbal. Algo realmente impactante en el uso de esta técnica con los TEA fue la curiosidad que causó en ellos el poder manipular los títeres, los cuales podían ser animados y movilizados por ellos mismos, lo que les permitía hacer monólogos y diálogos con sus compañeros, con diferentes respuestas y tonalidades de voz.

Es así como se evidenció que los jóvenes con TEA que no lograban expresar de forma verbal sus emociones y sentimientos, empezaron a hacerlo por medio de los títeres, a la vez que fueron mejorando sus niveles de interacción y comunicación con su entorno; esto último se hizo visible en el cierre del taller con la presentación de una obra a la comunidad del colegio sobre los cuidados del medio ambiente que mostró los avances en la comunicación e interacción social logrados a familiares y docentes, quienes confirmaron con satisfacción los avances en la comunicación de los TEA. 


\section{Pintura}

Cada participante se asumió como sujeto y pintó lo que sentía en su interior. La imitación del mundo visible cedió el paso a la expresión de los sentimientos. De esta manera pudimos evidenciar que estos jóvenes TEA a pesar de no hablar mucho, podían plasmar sus gustos, preferencias, de acuerdo con su estado emocional.

De otra parte, la experiencia que nos quedó como terapeutas es que definitivamente la pintura tiene una gran ventaja a nivel terapéutico en aquellas personas que presentan dificultad de comunicación a nivel de expresión verbal y no verbal, porque logran trasmitir de esta manera sensaciones y emociones reprimidas.

Tabla 2. Observación del desempeño individual en cada actividad artística

\begin{tabular}{|c|c|c|c|}
\hline TECNICA & CASO 1 & CASO 2 & CASO 3 \\
\hline Música & $\begin{array}{l}\text { En el caso linicialmente } \\
\text { se muestra un poco indife- } \\
\text { rente a la música, por el } \\
\text { hecho de que no le gusta } \\
\text { estar con el grupo; sin em- } \\
\text { bargo, le gusta el piano y } \\
\text { algunas canciones en es- } \\
\text { pecial, por lo que a partir } \\
\text { del gusto por el canto se le } \\
\text { incluyó en la coral y hay } \\
\text { dos canciones que canta } \\
\text { con mucha fuerza (a punto } \\
\text { de que las grita). } \\
\text { Por lo menos ya permite, } \\
\text { previo trabajo en equipo. }\end{array}$ & $\begin{array}{l}\text { Al comienzo se muestra un } \\
\text { poco tímido para integrarse } \\
\text { al grupo; sin embargo, al } \\
\text { ver compañeros de su ante- } \\
\text { rior colegio se anima y se } \\
\text { acerca a saludarlos. El } \\
\text { agrado y la fluidez por la } \\
\text { música es bastante notoria, } \\
\text { pues tiene excelente dispo- } \\
\text { sición para cantar; desarro- } \\
\text { lló videos musicales graba- } \\
\text { dos por él mismo, pues } \\
\text { maneja bastante los equi- } \\
\text { pos de cómputo y en ellos } \\
\text { experimenta mucho con el } \\
\text { diseño de los videos. }\end{array}$ & $\begin{array}{l}\text { Aunque se muestra bas- } \\
\text { tante apático a las reunio- } \\
\text { nes con sus compañeros, } \\
\text { tiene gran agilidad para } \\
\text { interpretar el piano, y los } \\
\text { instrumentos de percu- } \\
\text { sión; asimismo, con la } \\
\text { práctica de los talleres de } \\
\text { música ha mejorado su } \\
\text { manejo de voz. }\end{array}$ \\
\hline Danza & $\begin{array}{l}\text { En este caso el contacto per- } \\
\text { sonal y el trabajo en grupo } \\
\text { no le gustan; sin embargo, } \\
\text { se ha notado que ha empe- } \\
\text { zado a asociar la música con } \\
\text { el movimiento, y aunque al } \\
\text { principio no bailaba, le gus- } \\
\text { ta la música, por lo que se } \\
\text { integra y se deja tomar de la } \\
\text { mano para bailar. Con el } \\
\text { paso de los meses ha mejo- } \\
\text { rado su interacción con los } \\
\text { demás y su creatividad, y ha } \\
\text { empezado a hacer chistes. } \\
\text { Antes no permitía que se le } \\
\text { tomaran fotos, pero en el úl- } \\
\text { timo trimestre ya permite } \\
\text { que se tomen las fotos, aun- } \\
\text { que no permite los videos. }\end{array}$ & $\begin{array}{l}\text { En este caso le gusta bailar } \\
\text { y con la misma agilidad } \\
\text { que canta baila; tiene bue- } \\
\text { na disposición para bailar, } \\
\text { pero es muy acelerado y le } \\
\text { gusta dirigir y dar los co- } \\
\text { mandos (las órdenes) en } \\
\text { las danzas. }\end{array}$ & $\begin{array}{l}\text { En este caso el contacto } \\
\text { personal no le agrada mu- } \\
\text { cho, pero tiene disciplina y } \\
\text { comprende que debe bai- } \\
\text { lar en grupo, para que la } \\
\text { obra salga bien. Tiende a } \\
\text { repetir palabras y presenta } \\
\text { ecolalia en el momento de } \\
\text { escuchar los comandos del } \\
\text { instructor. Le encanta el } \\
\text { joropo y asume el lideraz- } \\
\text { go cuando se le da la or- } \\
\text { den; sin embargo, tiene } \\
\text { dificultades para memori- } \\
\text { zar los pasos. }\end{array}$ \\
\hline
\end{tabular}




\begin{tabular}{|c|c|c|c|}
\hline Títeres & $\begin{array}{l}\text { Al inicio muestra poca co- } \\
\text { municación con sus com- } \\
\text { pañeros ya que presenta } \\
\text { dificultad para expresarse; } \\
\text { sin embargo, lo que se ha } \\
\text { visto es que ve en los títe- } \\
\text { res amigos imaginarios. } \\
\text { Por lo tanto, solo habla } \\
\text { con ellos y por este medio } \\
\text { expresa emociones y sen- } \\
\text { saciones a los demás, que } \\
\text { no suele expresar en la co- } \\
\text { tidianidad. Se desinhibe } \\
\text { con los títeres y empieza } \\
\text { dialogar cambiando las } \\
\text { voces y entonaciones. }\end{array}$ & $\begin{array}{l}\text { Participa de forma muy } \\
\text { activa en cada uno de los } \\
\text { montajes coreográficos; } \\
\text { siempre quiere ser el rey el } \\
\text { que manda a todos, es bas- } \\
\text { tante saludable con todo el } \\
\text { mundo, pero lo hace por } \\
\text { repetición y si ve saludar } \\
\text { saluda a todos los que pa- } \\
\text { san por su lado. }\end{array}$ & $\begin{array}{l}\text { Inicialmente no tiene una } \\
\text { comunicación directa con } \\
\text { sus compañeros de grupo; } \\
\text { sin embargo, se le ha lo- } \\
\text { grado identificar una bue- } \\
\text { na capacidad de retórica a } \\
\text { través de los guiones que } \\
\text { se aprende para las obras } \\
\text { de teatro con los títeres. } \\
\text { Tiene excelente memoria } \\
\text { y creatividad, y modula la } \\
\text { voz de acuerdo con el per- } \\
\text { sonaje. Ha venido presen- } \\
\text { tado bastantes avances en } \\
\text { la interacción con otros } \\
\text { mediante esta actividad. }\end{array}$ \\
\hline Pintura & $\begin{array}{l}\text { Bastante disposición para } \\
\text { la pintura; sin embargo, } \\
\text { las manualidades en por- } \\
\text { celanicron y plastilina le } \\
\text { agradan más. Realiza } \\
\text { obras muy coloridas, pero } \\
\text { poco elaboradas. } \\
\text { Disciplinado, constante y } \\
\text { hasta que no termina no se } \\
\text { levanta. Relaciona los } \\
\text { colores con las frutas y } \\
\text { empezó a tomar iniciativa. }\end{array}$ & $\begin{array}{l}\text { Le agrada pintar y de he- } \\
\text { cho tiene muy buena dis- } \\
\text { posición, aunque siempre } \\
\text { hace las cosas con mucho } \\
\text { acelere por ende son muy } \\
\text { poco elaboradas, pues está } \\
\text { haciendo varias cosas a la } \\
\text { vez tiene atención disper- } \\
\text { sa. }\end{array}$ & $\begin{array}{l}\text { Bastante disposición para } \\
\text { la pintura, le gusta pintar } \\
\text { carros de fórmula } 1 \text {. } \\
\text { En este sentido es poco } \\
\text { creativo, por lo tanto, se le } \\
\text { deben poner patrones para } \\
\text { él pintarlos pero lo hace } \\
\text { muy bien y tiene memoria } \\
\text { fotográfica. }\end{array}$ \\
\hline
\end{tabular}

Fuente: elaboración propia.

\subsection{CAMBIOS EN LOS PARTICIPANTES CON TEA POR CATEGORÍAS}

Después de analizar las matrices donde se consignaron las observaciones periódicas de los participantes con TEA, así como las impresiones de los padres, se hizo una consolidación de resultados para determinar cuáles son los cambios en las destrezas comunicativas que adquirieron los jóvenes con TEA a través de las actividades artísticas, así como determinar cuáles son los cambios en su interacción social (Tabla 3). 
Tabla 3. Síntesis de cambios reportados por categorías

\begin{tabular}{|c|c|c|c|}
\hline \multicolumn{4}{|c|}{ Categoría 1. Habilidades de Comunicación } \\
\hline & Caso 1 & Caso 2 & Caso 3 \\
\hline $\begin{array}{l}\text { Conducta } \\
\text { Verbal }\end{array}$ & $\begin{array}{l}\text { Su comunicación verbal ha } \\
\text { mejorado, ya que establece } \\
\text { una comunicación más per- } \\
\text { manente con su madre y } \\
\text { con las personas más alle- } \\
\text { gadas de la escuela de artes, } \\
\text { pues ya se expresa mejor } \\
\text { sin gritar al hacerlo y al leer } \\
\text { señala lo que le llama la } \\
\text { atención, lo que le ha per- } \\
\text { mitido mejorar su nivel de } \\
\text { concentración y luego repe- } \\
\text { tir la información de inte- } \\
\text { rés. También ha mejorado } \\
\text { en el saludo a varias perso- } \\
\text { nas. }\end{array}$ & $\begin{array}{l}\text { Ha mejorado su nivel de } \\
\text { atención, socialización y } \\
\text { comunicación; la expresión } \\
\text { verbal ha mejorado, habla } \\
\text { con mayor fluidez, ya no se } \\
\text { tapa la boca para hablar y } \\
\text { se le escucha más claro; } \\
\text { ahora establece conversa- } \\
\text { ciones más tranquilas con } \\
\text { sus compañeros del grupo } \\
\text { de artes y en los ensayos de } \\
\text { danza y música. Es el que } \\
\text { lidera el grupo, pues dice } \\
\text { que él es el rey. }\end{array}$ & $\begin{array}{l}\text { La expresión verbal ha me- } \\
\text { jorado; ha disminuido el } \\
\text { mutismo selectivo, pues ya } \\
\text { pronuncia algunas frases y } \\
\text { saluda a las personas con } \\
\text { las que se relaciona. Sigue } \\
\text { presentando características } \\
\text { comunicativas monosíla- } \\
\text { bas, habla solo y responde } \\
\text { solo a lo que se le pregunta } \\
\text { sin agregar ningún comen- } \\
\text { tario; en ocasiones canta en } \\
\text { el hogar, y dialoga un poco } \\
\text { más con su hermana y con } \\
\text { su madre, situación bastan- } \\
\text { te diferente a cuando ingre- } \\
\text { só al programa. }\end{array}$ \\
\hline $\begin{array}{l}\text { Conducta no } \\
\text { verbal }\end{array}$ & $\begin{array}{l}\text { Tiene una evolución impor- } \\
\text { tante a nivel conductual y } \\
\text { han disminuido sus movi- } \\
\text { mientos estereotipados; } \\
\text { han bajado sus niveles de } \\
\text { ansiedad y ha mejorado no- } \\
\text { tablemente su comporta- } \\
\text { miento habitual al compar- } \\
\text { tir con otros compañeros. } \\
\text { Ya no se aísla de los demás, } \\
\text { pues permite que los otros } \\
\text { niños con discapacidad se } \\
\text { le acerquen y se han reduci- } \\
\text { do sus temores hacia los } \\
\text { demás, lo que le ha permiti- } \\
\text { do mejorar su nivel de co- } \\
\text { municación y convivencia } \\
\text { con otros. }\end{array}$ & $\begin{array}{l}\text { Sigue presentando episo- } \\
\text { dios de hiperactividad; } \\
\text { cuando se siente regañado o } \\
\text { cuando se le llama la aten- } \\
\text { ción, baja la cabeza y des- } \\
\text { vía la mirada, y a veces su } \\
\text { actitud frente al quehacer } \\
\text { dirigido por otras personas } \\
\text { se encuentra caracterizado } \\
\text { por la repetición de órdenes } \\
\text { para poder darle cumpli- } \\
\text { miento. }\end{array}$ & $\begin{array}{l}\text { Han disminuido sus movi- } \\
\text { mientos estereotipados, y } \\
\text { se han reducido significati- } \\
\text { vamente sus monólogos; } \\
\text { acata órdenes y comandos, } \\
\text { aunque en ocasiones suele } \\
\text { repetir las órdenes para sus } \\
\text { compañeros a fin de no co- } \\
\text { meter errores en los monta- } \\
\text { jes coreográficos de las } \\
\text { danzas. Sigue manifestan- } \\
\text { do obsesión por el orden de } \\
\text { las cosas y por mantenerlas } \\
\text { en el mismo lugar. Ha me- } \\
\text { jorado su nivel de atención } \\
\text { en tareas asignadas y se ha } \\
\text { incrementado su participa- } \\
\text { ción activa en las obras ar- } \\
\text { tísticas (títeres y danza). }\end{array}$ \\
\hline
\end{tabular}




\begin{tabular}{|c|c|c|c|}
\hline \multicolumn{4}{|c|}{ Categoría 2. Relaciones familiares y sociales } \\
\hline $\begin{array}{l}\text { Construcción } \\
\text { de relaciones } \\
\text { interpersonales } \\
\text { funcionales }\end{array}$ & $\begin{array}{l}\text { Ha mejorado su relación } \\
\text { con amigos y familiares; } \\
\text { han disminuido sus reac- } \\
\text { ciones de hipersensibilidad, } \\
\text { lo cual le ha permitido esta- } \\
\text { blecer nuevos canales de } \\
\text { comunicación con amigos } \\
\text { de la universidad y la es- } \\
\text { cuela de artes. Ha comen- } \\
\text { zado a emitir mensajes muy } \\
\text { personales y familiares, y la } \\
\text { estimulación con los títeres } \\
\text { le ha permitido mejorar su } \\
\text { tono de voz y su participa- } \\
\text { ción. }\end{array}$ & $\begin{array}{l}\text { Presenta cambios impor- } \\
\text { tantes a nivel de interacción } \\
\text { social y en sus relaciones } \\
\text { familiares. Ha aumentado } \\
\text { el nivel de comunicación a } \\
\text { través de las redes sociales; } \\
\text { con sus amigos, conocidos } \\
\text { y familiares, expresa de- } \\
\text { seos, preguntas, intereses } \\
\text { comunes. Su proceso de } \\
\text { formación artística ha me- } \\
\text { jorado, logrando mayor vi- } \\
\text { sibilización en los medios } \\
\text { de comunicación; atiende } \\
\text { entrevistas muy particula- } \\
\text { res, ya que quienes lo cono- } \\
\text { cen por su discapacidad lo } \\
\text { quieren entrevistar por su } \\
\text { gran talento musical. Ha } \\
\text { generado bastante admira- } \\
\text { ción y respeto; el con orgu- } \\
\text { llo dice "sí, yo soy el rey". }\end{array}$ & $\begin{array}{l}\text { Muestra mejoría a nivel de } \\
\text { socialización e interacción } \\
\text { con su familia y amigos; } \\
\text { demuestra iniciativa en } \\
\text { casa y ya sale a la iglesia y } \\
\text { saluda a las personas que } \\
\text { entran allí; aunque no per- } \\
\text { mite que se acerquen, él } \\
\text { saluda de forma muy corta } \\
\text { y cada vez que lo saludan y } \\
\text { le preguntan cómo está él, } \\
\text { dice "bien gracias". }\end{array}$ \\
\hline $\begin{array}{l}\text { Integración e } \\
\text { i n te r a c c ión } \\
\text { efectiva }\end{array}$ & $\begin{array}{l}\text { Participa más de las activi- } \\
\text { dades. Ha mejorado en nor- } \\
\text { mas de cortesía sus niveles } \\
\text { de interacción e integración } \\
\text { familiar y social han mejo- } \\
\text { rado, lo que le ha permitido } \\
\text { bajar sus niveles de ansie- } \\
\text { dad y agresividad. }\end{array}$ & $\begin{array}{l}\text { Sus niveles de integración e } \\
\text { interacción a nivel familiar } \\
\text { escolar se han fortalecido } \\
\text { notablemente, reforzando } \\
\text { bastante los niveles de aten- } \\
\text { ción y concentración en el } \\
\text { desarrollo de tareas, lo que } \\
\text { le ha permitido llevar una } \\
\text { relación afectiva armónica } \\
\text { con su grupo familiar, } \\
\text { Avanzó también en el se- } \\
\text { guimiento de normas en } \\
\text { casa y socialización en co- } \\
\text { munidad; el proceso le ha } \\
\text { permitido mejorar su nivel } \\
\text { de interacción con los me- } \\
\text { dios de comunicación, que } \\
\text { se han interesado en este } \\
\text { joven por su talento musi- } \\
\text { cal. }\end{array}$ & $\begin{array}{l}\text { Su nivel de integración e } \\
\text { interacción ha mejorado } \\
\text { bastante, lo cual le ha per- } \\
\text { mitido lograr una armonía } \\
\text { familiar más activa, situa- } \\
\text { ción que se corrobora en el } \\
\text { reconocimiento que hace la } \\
\text { madre del joven, quien } \\
\text { agradece permanentemente } \\
\text { en la escuela de artes el } \\
\text { apoyo recibido tanto para el } \\
\text { joven como para la familia. }\end{array}$ \\
\hline
\end{tabular}


Después de analizar cada caso, se puede dar como resultado global lo siguiente: se evidenció que el impacto del arte como herramienta comunicacional se dio en mejorar el nivel de atención, imaginación y creatividad, a la vez que permitió reformar sus representaciones simbólicas; igualmente, hay un avance en la expresión verbal y no verbal de los jóvenes, mejorando con ello su desarrollo afectivo, cognitivo y social. Como aspecto a considerar, los resultados del análisis cualitativo indican que, a pesar de que se notaron cambios en todos los casos, las características sintomáticas específicas en algunos de los casos no permitió el avance esperado del participante según los objetivos establecidos. Igualmente, los padres reportaron que durante el periodo de receso académico, los tres jóvenes tuvieron una disminución de los cambios positivos logrados durante el periodo escolar y mostraron retrocesos en las habilidades comunicativas y sociales.

Por categorías en síntesis se observa lo siguiente:

\subsubsection{Categoría 1: Desarrollo de habilidades de comunicación de los TEA}

En los 3 casos los participantes con TEA presentan una importante evolución a nivel de interacción y habilidades comunicativas. Con el apoyo del arte como herramienta pedagógica e inclusiva, logrando mejorar sus habilidades de comunicación funcional y cognitiva. Es evidente que el desarrollo de las habilidades de comunicación de los jóvenes con TEA requiere de un especial apoyo y seguimiento en su entorno social habitual.

\subsubsection{Categoría 2: Relaciones familiares y sociales de los TEA}

En esta categoría encontramos que la mayoría de estas familias con presencia de hijos con TEA había tenido problemas por la falta de un manejo adecuado de la patología y de las pautas de crianza, lo cual había repercutido en conflictos y actitudes de agresividad o aislamiento de los TEA; como resultados de los talleres de arte se evidencia que los participantes han disminuido las actitudes de agresividad o las manifestaciones de ansiedad con sus familiares.

\section{DISCUSIÓN Y CONCLUSIONES}

Un primer resultado del presente estudio fue el poder concluir que hubo avances en las habilidades de comunicación e interacción social en los jóvenes diagnosticados con TEA, cuando se utilizan actividades artísticas como herramientas pedagógicas y didácticas. Esto lleva a corroborar que la danza, la música, los títeres y la pintura son una alternativa para reforzar los logros de las terapias tradicionalmente utilizadas en este tipo de trastornos, las cuales aún no demuestran una efectividad comprobada (Mebarak, Martínez \& Serna, 2009); así, el arte funcionó como un método pedagógico que utiliza formas de expresión y comprensión que pueden ser más efectivas que las tradicionales. Se puede decir también que el mejoramiento de las habilidades de interacción y comunicación social de los jóvenes con TEA demuestra que los potenciales de desarrollo social y comunicativo en personas con TEA pueden expandirse más allá de lo que puede sugerir el diagnóstico, dependiendo claro está, de la severidad de los síntomas; este resultado se puede relacionar con las afirmaciones de Romañach y Palacios (2008), acerca de que las personas con discapacidad 
no carecen de capacidades, sino que funcionan de una manera que no es la habitual; por lo tanto, con los medios y apoyos adecuados, pueden desarrollar muchas de las funciones que desempeña cualquier persona. Por lo tanto, el diseño y aplicación de estrategias y modos distintos, acordes con estos funcionamientos diversos, puede lograr avances en la comunicación y la interacción social, capacidades habitualmente comprometidas en el TEA. Asimismo, se pudo observar que las actividades artísticas lograron estimular procesos anexos que dificultan el desarrollo de las habilidades de comunicación e interacción, tales como la comprensión y diferenciación entre el mundo interno y el mundo externo de los participantes de los talleres o la modulación de emociones e impulsos, confirmando hallazgos de diversas investigaciones (Arce, 2008; Benavides \& Orrego, 2010; Epelde, 2009; Freundlich, Pike \& Schwartz, 1989; Moghaddam et al., 2008).

Del mismo modo, se evidenció en la población objeto de estudio un mejoramiento a nivel de atención y creatividad; Igualmente, el estudio permitió identificar que el arte refuerza sus representaciones simbólicas, optimizando su expresión verbal y no verbal, aptitudes necesarias para salir del aislamiento del espectro autista.

De otra parte, se logró evidenciar que, durante el periodo de receso académico, los jóvenes tuvieron una disminución de los cambios positivos logrados en sus actividades diarias durante el periodo escolar, y mostraron una disminución en las habilidades comunicativas y sociales. Esta situación permite evidenciar que se requiere de un trabajo sostenido en el tiempo, a fin evitar retrocesos (López \& Rivas, 2014).

Como limitaciones del estudio, este se enfocó en participantes con una edad específica, entre 25 y 27 años, sin contemplar diferencias de género, condición socioeconómica, tipo o grado de la sintomatología, por lo que no se pueden hacer inferencias al respecto. Igualmente, el estudio se centró en la capacidad del arte como método pedagógico, pero se recomienda también un estudio más centrado en los efectos de distintas técnicas asociadas al arte, para poder avanzar en este método como una intervención pedagógica para esta población específica. Igualmente, el método cualitativo etnográfico no tiene el propósito de ser repetible o generalizable en sus resultados, los cuales están sujetos a las características contextuales, a las diferencias personales tanto de los participantes como de los observadores y las particularidades socioculturales del grupo estudiado. De otra parte, por la misma condición del método utilizado, tanto las actividades como los efectos a observar son procesos abiertos, emergentes, guiados por categorías semi-estructuradas más que estandarizados o tipificados, pues tienen como fin reconocer en cada caso sus cambios particulares, lo que hace que los resultados en cada caso no permitan hacer predicciones concretas sobre futuras réplicas de esta experiencia. Además, se reconoce que otros aspectos externos a la investigación pueden incidir en los cambios reportados a lo largo del periodo de aplicación de los talleres. Por lo tanto, el aporte está en ver los efectos y las diferencias en el grupo estudiado al atravesar por este método pedagógico, más que en demostrar que los cambios se deban exclusivamente al mismo.

\section{REFERENCIAS BIBLIOGRÁFICAS}

Angrosino, M. (2012). Etnografía y observación participante en investigación cualitativa. Madrid: Morata.

Arce, M. (2008). Soluciones pedagógicas para el Autismo. Qué hacer con los TGD. España: Ariednal 
S.R.L.

Artigas-Pallares, J., \& Paula, I. (2012). El autismo 70 años después de Leo Kanner y Hans Asperger. Revista de la Asociación Española de Neuropsiquiatría, 32(115), 567-587. doi:10.4321/S021157352012000300008

Baron-Cohen, S. (1990). Autism: A Specific Cognitive Disorder of \& 1squo; Mind-Blindness'. International Review of Psychiatry, 2(1), 81-90.

Barquero M. S. (2008). Música y Cerebro. Circunvalación del Hipocampo. Recuperado desde http:// www.hipocampo.org/originales/original0002.asp

Benavides, H., \& Orrego, P. (2010). Música en un niño con trastorno de espectro de autismo. Revista de la Asociación Española de Neuropsiquiatría, 147-153.

Betés del Toro, M. (2000). Fundamentos de la Musicoterapia. Madrid: Ediciones Morata.

Brun, J., \& Villanueva, R. (2004). Niños con Autismo. Experiencia y Experiencias. Valencia: Promolibro.

Cuxart, F. (2000). El autismo: aspectos descriptivos y terapéuticos. Málaga: Aljibe Archidona.

Epelde, E. (2009). Terapia por la pintura y psicoanálisis. Avances en Salud Mental Relacional, 8(2), $1-7$.

Freundlich, B., Pike, L., \& Schwartz, V. (1989). Dance and Music for Children with Autism. The Journal of Physical Education, 60(9), 50-53.

García, J., \& Peñas, J. (2009). Autismo, epilepsia y patología del lóbulo temporal, Neurología, 48(Supl 2), S35-S45.

Hernández, R., Fernández-Collado, C., \& Baptista, P. (2007). Metodología de la investigación (4a ed.). México: Mc Graw-Hill.

Joseph, R. M., Tager-Flusberg, H., \& Lord, C. (2002). Cognitive profiles and social-communicative functioning in children with autism spectrum disorder. Journal of Child Psychology and Psychiatry and Allied Disciplines, 43(6), 807-821.

Lions, M. (s. f.). Association Marionnette et Thérapie: La elaboración de un fenómeno. Recuperado de: http://www.teiamoner.com/llicencia/formacioE.htm

López, M., \& González, M. (2003). Inteligencia emocional, habilidad social, control de las emociones, destreza interpersonal, seguridad interior. Bogotá: Gama.

López, S., \& Rivas, R. (2014). El trastorno del espectro del autismo: Retos, oportunidades y necesidades. Informes Psicológicos, 14(2), 13-31.

Martín, M. (2009). Art Therapy and Autism: Overview and Recommendations. Art Therapy, 26(4), 187-190. doi:10.1080/07421656.2009.10129616

Martos, J., \& Ayuda, R. (2002). Comunicación y lenguaje en el espectro autista: el autismo y la disfasia. Revista de neurología, 34, S58-S63.

Mebarak, M., Martínez, M., \& Serna, A. (2009). Revisión bibliográfica analítica acerca de las diversas teorías y programas de intervención del autismo infantil. Psicología desde el Caribe, $24,120-146$.

Miguel A. (2006). El Mundo de las emociones en los autistas. Teoría de la Educación y Cultura en la Sociedad de la Información, 7(2),169-183.

Ministerio de Educación de Chile. (2010). Educación de estudiantes que presentan trastornos del espectro autista. Santiago de Chile: Autor.

Moghaddam, K., Zadeh, A., Sharifi, P., \& Afrooz, G. (2008). Effect of the family-based art therapy program on the social interactions, verbal Skills and stereotypic behaviors of Children with Autism Spectrum Disorders (ASD). Iranian Journal of Public Health, 45(6), 830-832.

Morrison, J. (2015). DSM-5: guía para el diagnóstico clínico. México: Manual Moderno.

Organización Mundial de la Salud [OMS]. (2006). CIE 10, trastornos mentales y del comportamiento: descripciones clínicas y pautas para el diagnóstico. Madrid: Meditor.

Rajendran, G., \& Mitchell, P. (2007). Cognitive theories of autism. Developmental Review, 27, 224226. doi:10.1016/j.dr.2007.02.001 
Rodríguez,A. C., \& Rodríguez, M. A. (2002). Diagnóstico clínico del autismo. Revista de Neurología, 34, $72-77$.

Romañach, J., \& Palacios, A. (2008). El modelo de la diversidad: una nueva visión de la bioética desde la perspectiva de las personas con diversidad funcional (discapacidad). Intersticios: revista sociológica de pensamiento crítico, 2(2), 37-47.

Russell, J. (2000). El autismo como trastorno de la función ejecutiva. Madrid: Editorial Panamericana. Sperber, D., \& Wilson, D. (1986). Relevance: communication and cognition. Oxford: Basil Blackwell.

Tager-Flusberhg, H. (1989). An analysis of discourse ability and internal state lexicons in a longitudinal study of autisic children. Paper presented at the SRCD, Kansas City, Retrieve from: http://www.researchgate.net/publication/232042718_Autism_A_Specific_Cognitive_Disorder_ of_lsquoMind-Blindness/file/3deec5173ac60c133e.pdf

Talero. C., Martínez, L., Mercado, M., Ovalle, J., Velásquez, A., \& Zarruk, J. (2003). Autismo, Estado del Arte. Ciencias de la Salud, 68-85.

Watzlawick, P., Beavin, J., \& Jackson, D. (1986). Teoría de la comunicación humana. Barcelona: Herder. 